\title{
The Solution of a Class of Singularly Perturbed Two-Point Boundary Value Problems by the Iterative Reproducing Kernel Method
}

\author{
Zhiyuan Li, ${ }^{1}$ YuLan Wang, ${ }^{1}$ Fugui Tan, ${ }^{2}$ \\ Xiaohui Wan, ${ }^{1}$ and Tingfang Nie ${ }^{1}$ \\ ${ }^{1}$ Department of Mathematics, Inner Mongolia University of Technology, Hohhot 010051, China \\ 2 Jining Teachers College, Jining 012000, China \\ Correspondence should be addressed to YuLan Wang, wylnei@163.com
}

Received 14 February 2012; Revised 7 April 2012; Accepted 18 April 2012

Academic Editor: Shaoyong Lai

Copyright (c) 2012 Zhiyuan Li et al. This is an open access article distributed under the Creative Commons Attribution License, which permits unrestricted use, distribution, and reproduction in any medium, provided the original work is properly cited.

In (Wang et al., 2011), we give an iterative reproducing kernel method (IRKM). The main contribution of this paper is to use an IRKM (Wang et al., 2011), in singular perturbation problems with boundary layers. Two numerical examples are studied to demonstrate the accuracy of the present method. Results obtained by the method indicate that the method is simple and effective.

\section{Introduction}

Singularly perturbed problems (SPPs) arise frequently in applications including geophysical fluid dynamics, oceanic and atmospheric circulation, chemical reactions, and optimal control. In this paper, we consider the following singularly perturbed two-point boundary value problem:

$$
\begin{gathered}
\varepsilon U_{x x}(x)+a(x) U_{x}(x)+b(x) U(x)=F(x, U(x)), \quad x \in(0,1), \\
U(0)=\alpha, \quad U(1)=\beta,
\end{gathered}
$$

where $\varepsilon$ is a positive small parameter, $a(x), b(x)$, and $F(x, v)$ are known functions, and $U(x)$ is a unknown function to be determined. In this paper, we assume that (1.1) has a unique solution that belongs to $W_{2}^{3}[0,1]$. Like in $[1-5]$, we give reproducing 
kernel spaces $W_{2}^{1}[a, b]$ and $W_{2}^{3}[a, b]$. (i) We define the inner product space $W_{2}^{1}[a, b]=$ $\left\{u \mid u\right.$ is one-variable absolutely continuous function, $\left.u^{\prime} \in L^{2}[a, b]\right\}$. The inner product is given by $\langle u(x), v(x)\rangle_{W_{2}^{1}}=u(a) v(a)+\int_{a}^{b} u^{\prime}(x) v^{\prime}(x) d x$. The space $W_{2}^{1}[a, b]$ is a reproducing kernel space, and its reproducing kernel is $R_{x}^{\{1\}}(y)$. (ii) Space $W_{2}^{3}[a, b]=$ $\left\{u \mid u, u^{\prime}, u^{\prime \prime}\right.$ is one-variable absolutely continuous function, $\left.u(a)=u(b)=0, u^{\prime \prime \prime} \in L^{2}[a, b]\right\}$.

The inner product is given by $\langle u(x), v(x)\rangle_{W_{2}^{3}}=u^{\prime}(a) v^{\prime}(a)+u^{\prime \prime}(a) v^{\prime \prime}(a)+$ $\int_{a}^{b} u^{\prime \prime \prime}(x) v^{\prime \prime \prime}(x) d x$. The space $W_{2}^{3}[a, b]$ is a reproducing kernel space, and its reproducing kernel is $R_{x}^{\{2\}}(y)$.

\section{Iterative Reproducing Kernel Method (IRKM)}

In order to solve (1.1), we first give the analytical and approximate solutions of the following operator equation:

$$
(L u)(x)=F(x, u(x))
$$

where $L: H[a, b] \rightarrow H_{1}[a, b]$ is a bounded linear operator and $L^{-1}$ is existent. $H_{1}[a, b]$ is an RKHS with the reproducing kernel $\bar{K}(x, t), H[a, b]$ is also an RKHS with the reproducing kernel $K(x, t)$.

Theorem 2.1. If $L^{-1}$ is existent and $\left\{x_{i}\right\}_{i=1}^{\infty}$ are countable dense points in $[a, b]$, Letting $\bar{\psi}_{i}(x)=$ $\sum_{k=1}^{i} \beta_{i k} \psi_{k}(x)$, where the $\beta_{i k}$ are the coefficients resulting from the Gram-Schmidt orthonormalization, $\psi_{i}(x)=\left(L_{y} K(x, y)\right)\left(x_{i}\right), i=1,2, \ldots$, then

$$
u(x)=\sum_{i=1}^{\infty} \sum_{k=1}^{i} \beta_{i k} f\left(x_{k}, u\left(x_{k}\right)\right) \bar{\psi}_{i}(x)
$$

is an analytical solution of (2.1).

Proof. $u(x)$ can be expanded to the Fourier series in terms of normal orthogonal basis $\left\{\bar{\psi}_{i}(x)\right\}_{i=1}^{\infty}$ in $H[a, b]:$

$$
\begin{aligned}
u(x) & =\sum_{i=1}^{\infty}\left\langle u(x), \bar{\psi}_{i}(x)\right\rangle \bar{\psi}_{i}(x) \\
& =\sum_{i=1}^{\infty} \sum_{k=1}^{i} \beta_{i k}\left\langle u(x), \psi_{k}(x)\right\rangle \bar{\psi}_{i}(x) \\
& =\sum_{i=1}^{\infty} \sum_{k=1}^{i} \beta_{i k}\left\langle u(x),\left(L_{s} K_{x}(s)\right)\left(x_{k}\right)\right\rangle \bar{\psi}_{i}(x) \\
& =\sum_{i=1}^{\infty} \sum_{k=1}^{i} \beta_{i k}\left(L_{s}\left\langle u(x), K_{x}(s)\right\rangle\right)\left(x_{k}\right) \bar{\psi}_{i}(x)
\end{aligned}
$$




$$
\begin{aligned}
& =\sum_{i=1}^{\infty} \sum_{k=1}^{i} \beta_{i k}\left(L_{s} u(s)\right)\left(x_{k}\right) \bar{\psi}_{i}(x) \\
& =\sum_{i=1}^{\infty} \sum_{k=1}^{i} \beta_{i k} f\left(x_{k}, u\left(x_{k}\right)\right) \bar{\psi}_{i}(x) .
\end{aligned}
$$

\section{(i) Linear Problem}

Suppose (2.1) is a linear problem, that is, $f(x, u)=F(x)$. We define an approximate solution $u_{n}(x)$ by

$$
u_{n}(x)=\sum_{i=1}^{n} \sum_{k=1}^{i} \beta_{i k} F\left(x_{k}\right) \bar{\psi}_{i}(x)
$$

Theorem 2.2 (convergence analysis). Let $\varepsilon_{n}^{2}=\left\|u(x)-u_{n}(x)\right\|^{2}$; then the sequence of real numbers $\varepsilon_{n}$ is monotonously decreasing and $\varepsilon_{n} \rightarrow 0$ and the sequence $u_{n}(x)$ is convergent uniformly to $u(x)$, $k=0,1,2$.

Proof. We have

$$
\varepsilon_{n}^{2}=\left\|u(x)-u_{n}(x)\right\|^{2}=\sum_{i=n+1}^{\infty}\left\langle u(x), \bar{\psi}_{i}(x)\right\rangle \bar{\psi}_{i}(x)=\sum_{i=n+1}^{\infty}\left(\left\langle u(x), \bar{\psi}_{i}(x)\right\rangle\right)^{2},
$$

and clearly $\varepsilon_{n-1} \geq \varepsilon_{n}$ and consequently $\left\{\varepsilon_{n}\right\}$ is monotone decreasing in the sense of $\|\cdot\|$. By Theorem 2.1, we know that $\sum_{i=1}^{\infty}\left\langle u(x), \bar{\psi}_{i}(x)\right\rangle \bar{\psi}_{i}(x)$ is convergent in the norm of $\|\cdot\|$, then we have $\varepsilon_{n}^{2}=\left\|u(x)-u_{n}(x)\right\|^{2} \rightarrow 0$.

For any $x \in[a, b], k=0,1,2$,

$$
\left|u_{n}^{(k)}(x)-u^{(k)}(x)\right|=\left|\left\langle u_{n}(t)-u(t), \frac{\partial^{k} K(x, t)}{\partial x^{k}}\right\rangle\right| \leq\left\|u_{n}(t)-u(t)\right\| \cdot\left\|\frac{\partial^{k} K(x, t)}{\partial x^{k}}\right\|,
$$

and by the expression of $K(x, t)$, there exists $C_{k}>0$, such that $\left\|\partial^{k} K(x, t) / \partial x^{k}\right\|<C_{k}$; thus

$$
\left|u_{n}^{(k)}(x)-u^{(k)}(x)\right| \leq C_{k}\left\|u_{n}(t)-u(t)\right\|=C_{k} \varepsilon_{n} \longrightarrow 0
$$

\section{(ii) Nonlinear Problem}

Suppose that (1.1) is a nonlinear problem, that is, $f(x, u)=N(u)+F(x)$, where $N: H[a, b] \rightarrow$ $H_{1}[a, b]$ is a nonlinear operator, and we give an iterative sequence $u_{n}(x)$ :

$$
\begin{aligned}
& u_{0, *}(x) \text { is the solution of the linear equation } L u=F(x), \\
& u_{n+1, *}(x) \text { is the solution of the linear equation } L u=N\left(u_{n, *}\right)+F(x), n=0,1,2 \ldots
\end{aligned}
$$


Lemma 2.3. If $u_{n, *}(x) \rightarrow u(x)$, then $u(x)$, is the solution of (1.1).

Theorem 2.4. Suppose that the nonlinear operator $A \triangleq\left(L^{-1} N\right): H_{1}[a, b] \rightarrow H[a, b]$ satisfies the contractive mapping principle, that is,

$$
\|A(u)-A(v)\| \leq \lambda\|u-v\|, \quad \lambda<1
$$

then $u_{n, *}(x)$ is convergent.

\section{Solution of Singularly Perturbed Problems}

We notice that a small variation in the parameter $\varepsilon$ produces a large variation in the solution. In other words, we are treating an ill-posed problem. In this paper, by dividing the domain $[0,1]$ into three subdomains $[0, d],[d, 1-d]$, and $[1-d, 1]$.

(i) Outer Region

We have

$$
\begin{gathered}
\varepsilon U_{x x}(x)+a(x) U_{x}(x)+b(x) U(x)=F(x, U(x)), \quad x \in[d, 1-d], \\
u(d)=p, \quad u(1-d)=q .
\end{gathered}
$$

Letting $\bar{u}(x)=U(x)-((x-d) /(1-2 d)) q+((x+d-1) /(1-2 d))$ and $\left(L_{1} \bar{u}\right)(x)=\varepsilon \bar{u}_{x x}(x)+$ $a(x) \bar{u}_{x}+b(x) \bar{u},(3.1)$ can further be converted into

$$
\left(L_{1} \bar{u}\right)(x)=\bar{f}(x, \bar{u}(x))
$$

where $\bar{f}(x, \bar{u}(x))=F(x, u(x))-a(x)((1 /(1-2 d)) q-(1 /(1-2 d)) p)-b(x)(((x-d) /(1-2 d)) q+$ $((x+d-1) /(1-2 d)) p)$. Using IRKM, we can get the solution of the outer region problem.

(ii) Left Layer

We have

$$
\begin{gathered}
\varepsilon U_{x x}(x)+a(x) U_{x}(x)+b(x) U(x)=F(x, U(x)), \quad x \in[0, d), \\
U(0)=\alpha, \quad U(d)=p \text { is known. }
\end{gathered}
$$

Letting $u(x)=U(x)-\alpha-(x / d)(p-\alpha), x / d=x_{1}$, then $x=d x_{1}, u(x)=u\left(d x_{1}\right) \doteq \bar{u}\left(x_{1}\right)$, $d u / d x=(1 / d)\left(d \bar{u} / d x_{1}\right)$, and $d^{2} u / d x^{2}=\left(1 / d^{2}\right)\left(d^{2} \bar{u} / d x_{1}^{2}\right)$. In space $W_{2}^{3}[0,1]$, (3.3) can further be converted into following form:

$$
\left(L_{\varepsilon} \bar{u}\right)\left(x_{1}\right)=\bar{f}\left(x_{1}, \bar{u}\left(x_{1}\right)\right),
$$

where $\bar{f}\left(x_{1}, \bar{u}\left(x_{1}\right)\right)=d^{2}(F(x, U(x))-(a(x) / d)(p-\alpha)-b(x)(\alpha+(x / d)(p-\alpha))),\left(L_{\varepsilon} \bar{u}\right)\left(x_{1}\right)=$ $\varepsilon \bar{u}_{x_{1} x_{1}}\left(x_{1}\right)+a\left(x_{1}\right) d \bar{u}_{x_{1}}\left(x_{1}\right)+b\left(x_{1}\right) d^{2} \bar{u}\left(x_{1}\right)$. Using IRKM, we can get the solution of the inner region (left layer near) problem. 
Table 1: Comparison of results, $x \in[0, d], \varepsilon=2^{-10}$, and $d=0.1$.

\begin{tabular}{lcccc}
\hline$x$ & $u_{T}(x)$ & $u_{100}(x)$ & $\left|u_{T}-u_{100}\right|$ & $\left|u_{T}-u_{200}\right|$ \\
\hline 0 & 0 & 0 & 0 & 0 \\
0.01 & -0.272864 & -0.272866 & $1.8788 \times 10^{-6}$ & $7.48551 \times 10^{-7}$ \\
0.02 & -0.468765 & -0.46877 & $5.38727 \times 10^{-6}$ & $1.15911 \times 10^{-7}$ \\
0.03 & -0.608251 & -0.608257 & $6.10734 \times 10^{-6}$ & $1.17267 \times 10^{-7}$ \\
0.04 & -0.706254 & -0.70626 & $5.54546 \times 10^{-6}$ & $1.01462 \times 10^{-7}$ \\
0.05 & -0.773632 & -0.773636 & $4.52354 \times 10^{-6}$ & $8.05672 \times 10^{-7}$ \\
0.06 & -0.818281 & -0.818285 & $3.45181 \times 10^{-6}$ & $6.04003 \times 10^{-7}$ \\
0.07 & -0.845955 & -0.845958 & $2.49497 \times 10^{-6}$ & $4.30798 \times 10^{-7}$ \\
0.08 & -0.860849 & -0.86085 & $1.66887 \times 10^{-6}$ & $2.84958 \times 10^{-7}$ \\
0.09 & -0.866029 & -0.86603 & $8.9114 \times 10^{-7}$ & $1.50666 \times 10^{-7}$ \\
0.1 & -0.863746 & -0.863746 & $3.33067 \times 10^{-16}$ & $2.22045 \times 10^{-7}$ \\
\hline
\end{tabular}

Table 2: Comparison of results, $x \in[d, 1-d], \varepsilon=2^{-30}$, and $d=0.001$.

\begin{tabular}{lcccc}
\hline$x$ & $u_{T}(x)$ & $u_{100}(x)$ & $\left|u_{T}-u_{10}\right|$ & $\left|u_{T}-u_{100}\right|$ \\
\hline 0.001 & -0.99999 & -0.99999 & $1.51212 \times 10^{-13}$ & $4.71756 \times 10^{-12}$ \\
0.1008 & -0.903026 & -0.903045 & $1.89196 \times 10^{-5}$ & $7.69723 \times 10^{-10}$ \\
0.2006 & -0.652715 & -0.652709 & $5.8384 \times 10^{-6}$ & $1.58393 \times 10^{-10}$ \\
0.3004 & -0.344297 & -0.344299 & $1.66259 \times 10^{-6}$ & $4.10425 \times 10^{-10}$ \\
0.4002 & -0.0951225 & -0.0951222 & $3.12214 \times 10^{-7}$ & $2.67338 \times 10^{-10}$ \\
0.5998 & -0.0951225 & -0.0951223 & $1.7619 \times 10^{-7}$ & $6.92579 \times 10^{-10}$ \\
0.6996 & -0.344297 & -0.344298 & $1.11205 \times 10^{-6}$ & $2.66992 \times 10^{-10}$ \\
0.7994 & -0.652715 & -0.652711 & $3.96364 \times 10^{-6}$ & $1.77961 \times 10^{-10}$ \\
0.8992 & -0.903026 & -0.903039 & $1.27772 \times 10^{-5}$ & $6.22121 \times 10^{-8}$ \\
0.999 & -0.99999 & -0.99999 & $6.67721 \times 10^{-12}$ & $1.91558 \times 10^{-12}$ \\
\hline
\end{tabular}

(iii) Right Layer

We have

$$
\begin{gathered}
\varepsilon U_{x x}(x)+a(x) U_{x}(x)+b(x) U(x)=F(x, U(x)), \quad x \in(1-d, 1], \\
U(1)=\beta, \quad U(1-d)=q .
\end{gathered}
$$

Letting $u(x)=U(x)-\beta+((x-1) / d)(q-\beta),(x / d)-(1 / d)+1=x_{1}$, then $x=d x_{1}+1-d$, $u(x)=u\left(d x_{1}+1-d\right) \doteq \bar{u}\left(x_{1}\right), d u / d x=(1 / d)\left(d \bar{u} / d x_{1}\right)$, and $d^{2} u / d x^{2}=\left(1 / d^{2}\right)\left(d^{2} \bar{u} / d x_{1}^{2}\right)$. In space $W_{2}^{3}[0,1],(3.5)$ can further be converted into following form:

$$
\left(L_{\varepsilon} \bar{u}\right)\left(x_{1}\right)=\bar{f}\left(x_{1}, \bar{u}\left(x_{1}\right)\right),
$$

where $f(x, u(x))=F(x, U(x))+(a(x) / d)(q-\beta)-b(x)(\beta-((x-1) / d)(q-\beta)), q$ is known (the outer solution has been given), $\bar{f}\left(x_{1}, \bar{u}\left(x_{1}\right)\right)=d^{2} f(x, u(x))$, and $\left(L_{\varepsilon} \bar{u}\right)\left(x_{1}\right)=\varepsilon \bar{u}_{x_{1} x_{1}}\left(x_{1}\right)+$ $a\left(x_{1}\right) d \bar{u}_{x_{1}}\left(x_{1}\right)+b\left(x_{1}\right) d^{2} \bar{u}\left(x_{1}\right)$. Using IRKM, we can get the solution of the inner region 
Table 3: Comparison of results, $x \in[1-d, 1], \varepsilon=2^{-10}$, and $d=0.01$.

\begin{tabular}{cccccc}
\hline$x$ & $u_{T}(x)$ & $u_{10}(x)$ & $\left|u_{T}-u_{10}\right|$ & $u_{200}(x)$ & $\left|u_{T}-u_{200}\right|$ \\
\hline 0.991 & -0.249439 & -0.249431 & $8.11552 \times 10^{-6}$ & -0.249439 & $2.72812 \times 10^{-9}$ \\
0.992 & -0.225227 & -0.225219 & $7.75753 \times 10^{-6}$ & -0.225227 & $3.72799 \times 10^{-9}$ \\
0.993 & -0.200201 & -0.200194 & $7.17326 \times 10^{-6}$ & -0.200201 & $4.18195 \times 10^{-9}$ \\
0.994 & -0.174338 & -0.174331 & $6.40087 \times 10^{-6}$ & -0.174338 & $4.18298 \times 10^{-9}$ \\
0.995 & -0.147609 & -0.147604 & $5.47897 \times 10^{-6}$ & -0.147609 & $3.82517 \times 10^{-9}$ \\
0.996 & -0.119989 & -0.119984 & $4.44655 \times 10^{-6}$ & -0.119989 & $3.20353 \times 10^{-9}$ \\
0.997 & -0.0914472 & -0.0914438 & $3.34288 \times 10^{-6}$ & -0.0914472 & $2.41376 \times 10^{-9}$ \\
0.998 & -0.0619555 & -0.0619533 & $2.20746 \times 10^{-6}$ & -0.0619555 & $1.55216 \times 10^{-9}$ \\
0.999 & -0.0314835 & -0.0314825 & $1.07993 \times 10^{-6}$ & -0.0314835 & $7.1531 \times 10^{-10}$ \\
1. & $-2.22045 \times 10^{-16}$ & $-3.62743 \times 10^{-17}$ & $1.8577 \times 10^{-16}$ & $-3.62852 \times 10^{-17}$ & $1.85759 \times 10^{-16}$ \\
\hline
\end{tabular}

Table 4: Comparison of results, $x \in[0, d]$, and $d=0.01$

\begin{tabular}{lcccccc}
\hline$x$ & $u_{T}(x)$ & $u_{3,10}(x)$ & $\left|u_{T}-u_{3,10}\right|$ & $u_{T}(x)$ & $u_{3,10}(x)$ & $\left|u_{T}-u_{3,10}\right|$ \\
& $\varepsilon=2^{-10}$ & $\varepsilon=2^{-10}$ & $\varepsilon=2^{-10}$ & $\varepsilon=2^{-5}$ & $\varepsilon=2^{-5}$ & $\varepsilon=2^{-5}$ \\
\hline 0.001 & 0.0324619 & 0.0324564 & $5.56236 \times 10^{-6}$ & 0.00628391 & 0.0062835 & $-4.09563 \times 10^{-7}$ \\
0.002 & 0.063871 & 0.0638658 & $5.18059 \times 10^{-6}$ & 0.0125208 & 0.0125204 & $-3.72485 \times 10^{-7}$ \\
0.003 & 0.0942614 & 0.0942567 & $4.67451 \times 10^{-6}$ & 0.0187109 & 0.0187106 & $-3.57056 \times 10^{-7}$ \\
0.004 & 0.123666 & 0.123662 & $4.20082 \times 10^{-6}$ & 0.0248545 & 0.0248541 & $-3.30969 \times 10^{-7}$ \\
0.005 & 0.152117 & 0.152113 & $3.72089 \times 10^{-6}$ & 0.0309517 & 0.0309514 & $-3.0024 \times 10^{-7}$ \\
0.006 & 0.179645 & 0.179642 & $3.23223 \times 10^{-6}$ & 0.0370029 & 0.0370026 & $-2.61703 \times 10^{-7}$ \\
0.007 & 0.20628 & 0.206277 & $2.78314 \times 10^{-6}$ & 0.0430082 & 0.043008 & $-2.14219 \times 10^{-7}$ \\
0.008 & 0.232051 & 0.232049 & $2.18254 \times 10^{-6}$ & 0.048968 & 0.0489678 & $-1.56195 \times 10^{-7}$ \\
0.009 & 0.256986 & 0.256984 & $2.14645 \times 10^{-6}$ & 0.0548823 & 0.0548822 & $-8.66593 \times 10^{-8}$ \\
\hline
\end{tabular}

(right layer near) problem. After solving the inner and outer region problems, we combine their solutions to obtain an approximate solution to the original problem (1.1) over the interval $0 \leq x \leq 1$.

\section{Numerical Examples}

Example 4.1. This example is from [6-8]:

$$
\begin{gathered}
-\varepsilon u_{x x}+u=f(x), \quad 0 \leq x \leq 1, \\
u(0)=0, \quad u(1)=0 .
\end{gathered}
$$

We determine $f(x)$ to get the true solution, the true solution $u_{T}(x)=\left(e^{(x-1) / \sqrt{\varepsilon}}+e^{-x / \sqrt{\varepsilon}}\right) /(1+$ $\left.e^{-1 / \sqrt{\varepsilon}}\right)-\cos ^{2}(\pi x)$. The numerical results are given in Tables 1,2 , and 3 . 
Example 4.2. Considering the following nonlinear singularly perturbed problem with boundary layers

$$
\begin{gathered}
\varepsilon u_{x x}+\frac{e^{x}}{x} u_{x}+e^{u}=f(x), \quad 0<x \leq 1, \\
u(0)=0, \quad u(1)=0
\end{gathered}
$$

we determine $f(x)$ to get the true solution, the true solution $u_{T}(x)=1+(x-1) e^{-x / \sqrt{\varepsilon}}-$ $x e^{(x-1) / \sqrt{\varepsilon}}$. The numerical results are given in Tables 3 and 4 .

\section{Conclusions}

In this paper, IRKM was employed successfully for solving a class of SPPs with boundary layers. The numerical results show that the present method is an accurate and reliable analytical technique for SPP with boundary layers.

\section{Acknowledgments}

The authors thank the reviewers for their valuable suggestions, which greatly improved the quality of the paper. This paper is supported by the Natural Science Foundation of Inner Mongolia (no. 2009MS0103) and the project of Inner Mongolia University of Technology (no. ZS201036).

\section{References}

[1] Y. L. Wang, Z. Y. Li, Y. Cao, and X. H. Wan, "A new method for solving a class of mixed boundary value problems with singular coefficient," Applied Mathematics and Computation, vol. 217, no. 6, pp. 2768-2772, 2010.

[2] Y.-1. Wang and L. Chao, "Using reproducing kernel for solving a class of partial differential equation with variable-coefficients," Applied Mathematics and Mechanics, vol. 29, no. 1, pp. 129-137, 2008.

[3] Z. Chen and Z.-j. Chen, "The exact solution of system of linear operator equations in reproducing kernel spaces," Applied Mathematics and Computation, vol. 203, no. 1, pp. 56-61, 2008.

[4] Y. Wang, T. Chaolu, and Z. Chen, "Using reproducing kernel for solving a class of singular weakly nonlinear boundary value problems," International Journal of Computer Mathematics, vol. 87, no. 1-3, pp. 367-380, 2010.

[5] Y. Wang, X. Cao, and X. Li, "A new method for solving singular fourth-order boundary value problems with mixed boundary conditions," Applied Mathematics and Computation, vol. 217, no. 18, pp. 7385-7390, 2011.

[6] R. K. Bawa and S. Natesan, "A computational method for self-adjoint singular perturbation problems using quintic spline," Computers \& Mathematics with Applications, vol. 50, no. 8-9, pp. 1371-1382, 2005.

[7] S. C. S. Rao and M. Kumar, "Exponential B-spline collocation method for self-adjoint singularly perturbed boundary value problems," Applied Numerical Mathematics, vol. 58, no. 10, pp. 1572-1581, 2008.

[8] D. Herceg and D. Herceg, "On a fourth-order finite-difference method for singularly perturbed boundary value problems," Applied Mathematics and Computation, vol. 203, no. 2, pp. 828-837, 2008. 


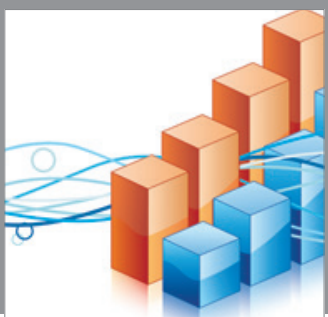

Advances in

Operations Research

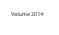

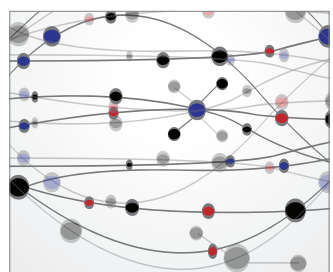

\section{The Scientific} World Journal
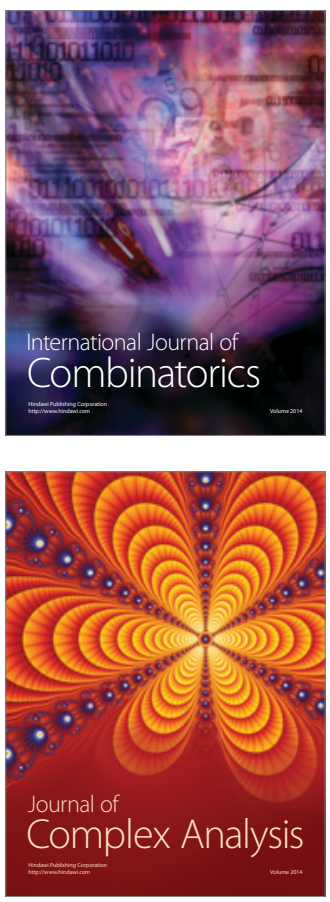

International Journal of

Mathematics and

Mathematical

Sciences
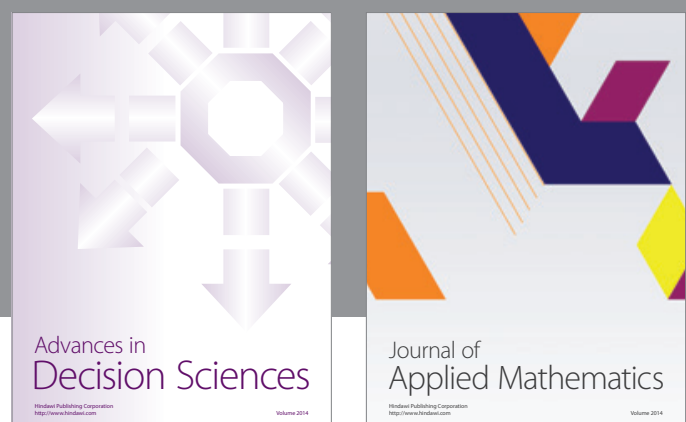

Journal of

Applied Mathematics
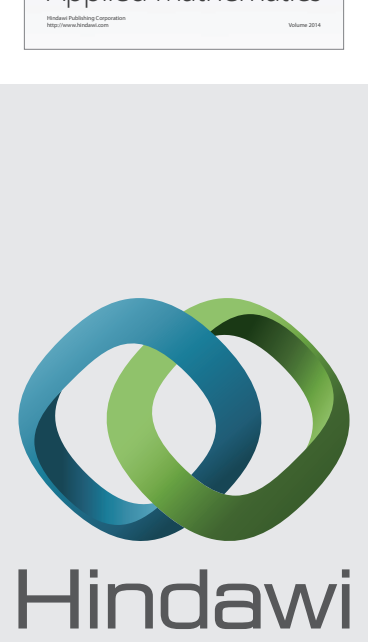

Submit your manuscripts at http://www.hindawi.com
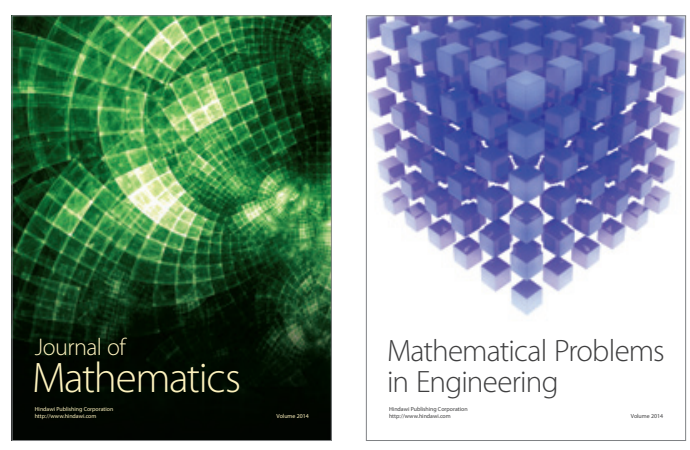

Mathematical Problems in Engineering
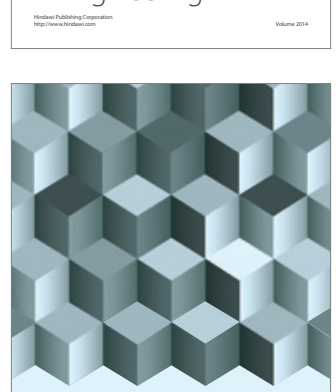

Journal of

Function Spaces
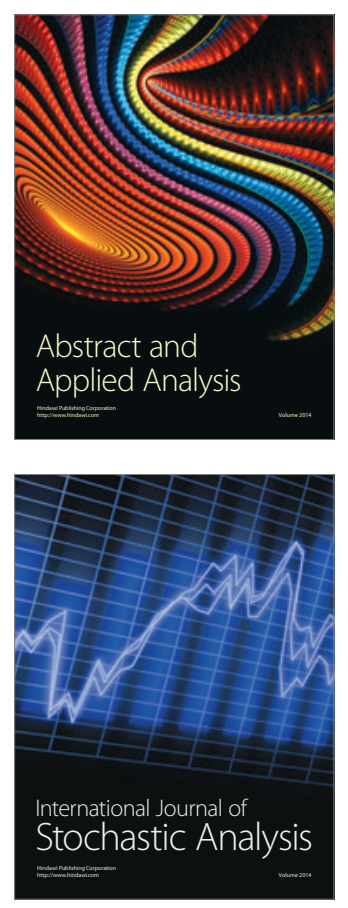

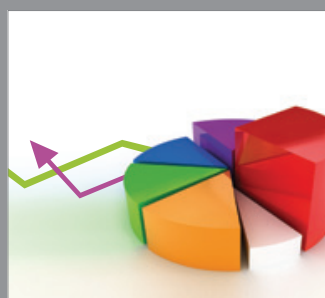

ournal of

Probability and Statistics

Promensencen
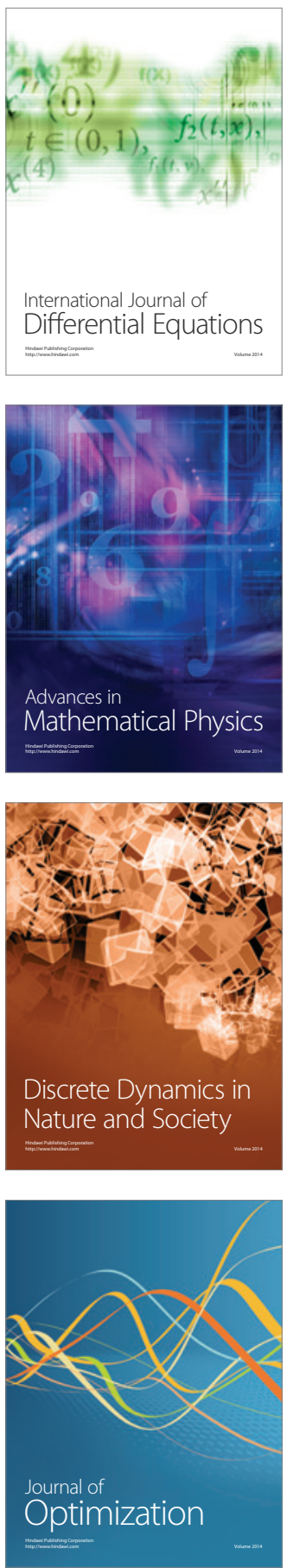